
\section{A perceptual learning study}

Nonnative phonemes are open to native interpretation:

Matthias J. Sjerps and James M. McQueen

Max Planck Institute for Psycholinguistics, Nijmegen, The Netherlands

\section{Introduction}

- Phonetic categories in one's native language are flexible: They can be altered by exposure to an ambiguous sound, presented in only 20 words (lexically-guided perceptual learning).

- Acquiring second-language (L2) phonetic categories is difficult, even after extensive exposure.

Can a (difficult) L2 phoneme be learnt to represent an L1 phoneme, when presented in L1 context?

- Four experiments investigated the possibilities and bounds of lexically-guided perceptual learning in speech perception, using (1) a digital /fs/-mixture, (2) English [ $[\theta$ ] (as in "bath"), (3) a nonspeech sound, and (4) natural instances of $/ \mathrm{f} /$ and $/ \mathrm{s} /$

\section{General methodology:}

\section{Cross-modal identity priming}

Training: Two groups of Dutch listeners were trained to interpret an ambiguous sound [?] as either /f/ or /s/, using /f/- or /s/-biasing words (e.g. "ongeloof" (disbelief) or "ingenieus" (ingenious)), which ended in [?] ("ongeloo?" or "ingenieu?").

Testing: We used Dutch minimal pairs like "doof'/"doos" (deaf/box). Listeners made visual lexical decisions to those words, after auditory primes. The primes were ambiguous versions of the minimal pairs (e.g. "doo?"), or unrelated words (control condition)

Training:
Gords (control condition).
Group1 1fl-final words, ending on [?]
ongeloo?
octaa?
..
.. (x 20)
Group 2 2 Is/-final words, ending on [?]
ingenieu?
paradij?
...$(\mathrm{x} 20)$

References:

McQueen, J. M., Cutler, A., \& Norris, D. (2006). Phonological
abstraction in the mental lexicon. Cognitive Science, 30, 1113-1126.

\section{Results}

\section{1. $[?]=/ \mathrm{fs} /$-mixture}

- Dutch listeners successfully learnt to interpret a digitally mixed /fs/-sound as representing either /f/ or /s/.

- Replication of McQueen et al. (2006).

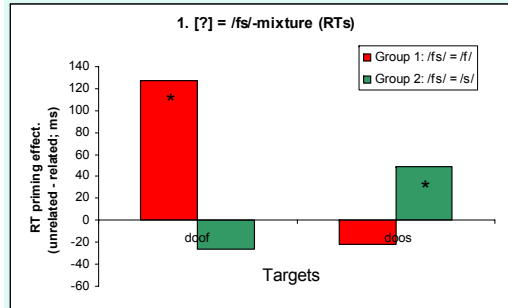

4. $[?]$ = natural fricatives

- Natural instances of [f] and [s] were used during "training"; one group at test heard [f]-final primes (e.g. "doof"), the other group heard [s]-final primes (e.g. "doos").

- This allowed for a comparison between the processing of "old" and "new" instances; and thus is a test of thoroughness of learning.

- The priming effects obtained with $[\theta]$ and /fs/ were very similar to the effects obtained with norma instances of /f/ and /s/.

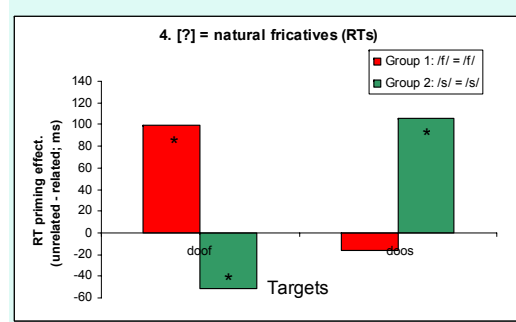

E-mail: Matthias.Sjerps@mpi.nl James.McQueen@mpi.nl

\section{2. $[?]=[\theta]$}

- Dutch listeners with English as L2 successfully learnt to interpret $[\theta]$ as representing /f/, or /s/, despite years of learning that $/ \mathrm{f} /, \mathrm{s} /$ and $[\theta]$ are distinct.

- Same amount of priming with $[\theta]$ as with /fs/.
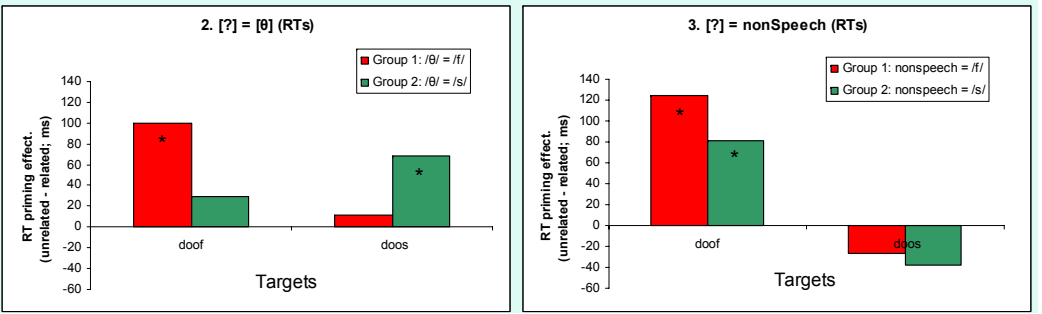

\section{Conclusions}

- English $[\theta]$ can be learnt to represent either /f/ or /s/ by L1 speakers of Dutch, when presented in L1 context.

- Perceptual learning of ambiguous items transfers to words that were not in the training set. This argues against a word-level episodic model of speech perception.

- The priming effects obtained with both /fs/ and $[\theta]$ were very similar to the effects obtained with natural fricatives. This finding reflects the fact that lexically-guided perceptual learning is fast and very thorough.

Perceptual learning in a native language is thorough, depends on spectral characteristics, and can override years of second-language phonetic learning. 